\title{
RESPUESTAS FISIOLÓGICAS DE VACAS HOLANDO ARGENTINO Y CRUZA JERSEY-HOLANDO, EN LA CUENCA LECHERA SANTAFESINA
}

\author{
LeVA, P. E. ${ }^{1}$; Garcia, M. S. ${ }^{1}$; Veles, M. A. ${ }^{1}$ \& Valtorta, S. E..$^{1,2}$
}

\section{RESUMEN}

Se compararon dos biotipos lecheros, Holstein $(\mathrm{H})$ y cruza Holstein - Jersey (CJ) para generar indicadores de adaptación al estrés térmico. El ensayo se efectúo en un tambo comercial entre el $14 / 1$ y el 28/2 de 2003. Se caracterizó el ambiente con el índice de temperatura y humedad (ITH) Se realizaron mediciones de temperatura rectal (TR), ritmo respiratorio (RR) y producción de leche. Para determinar el patrón de pastoreo se colocaron tacógrafos. Se compararon las diferencias entre las TR matutinas (TRm) y vespertinas y los RR matutinos (RRm) y vespertinos No se detectaron diferencias significativas entre biotipos. El efecto mes fue altamente significativo para TRm. No se detectó ningún efecto significativo en la producción de leche. El efecto mes fue significativo en la grasa. En el patrón de pastoreo no se presentaron diferencias si bien, $\mathrm{H}$ tiene un pastoreo nocturno de mayor duración. No se puede inferir un aumento en la tolerancia al estrés térmico para la cruza $(\mathrm{CJ})$.

Palabras clave: Holstein, Jersey, temperatura rectal; ritmo respiratorio; producción y composición de la leche, patrón de pastoreo.

\section{SUMMARY}

\section{Physiological and productive responses of two milking types}

Two milking types, Holstein (H) and H x Jersey cross (CJ) were compared to analyze physiological, productive and grazing time responses. The trial was performed in a commercial dairy farm from Jan $14^{\text {th }}$ till Feb $28^{\text {th }}, 2003$. The environment was characterized by measn of the temperature humidity index (THI). Rectal temperature (RT), respiration rate (RR) and milk production and composition were measured. Vibracordres were utilized to determine grazing time. Variations between morning and afternoon RT and respiration rate RR. No significant differences were recorded between milking types. A highly significant month effect was detected for morning RT. No signiicant effect was found

1.- Facultad de Ciencias Agrarias, Universidad Nacional del Litoral. Kreder 2805. (3080) Esperanza, provincia de Santa Fe. Telefax: (03496) 426400. E-mail: pleva@fca.unl.edu.ar

2.- Investigadora del Consejo Nacional de Investigaciones Científicas y Técnicas (CONICET).

Manuscrito recibido el 31 de marzo de 2005 y aceptado para su publicación el 9 de setiembre de 2005. 
for milk production. There was a significant month effect on milk fat. There were no differences in total grazing time. However, there was a trnd for $\mathrm{H}$ to graze longer during night hours. No creossderived improvement in heat tolerance could be detected.

Key words: Holstein, Jersey, Rectal temperature, Respiratory rate, Milk production and composition, grazing pattern. 